\title{
Choosing a Cut-Off on the Severity of Dependence Scale for Ecstasy Use
}

\author{
Raimondo Bruno*, Rapson Gomez and Allison Matthews
}

School of Psychology, University of Tasmania, Hobart, Tasmania, Australia

Keywords: Ecstasy, dependence, 3,4-methylenedioxymethamphetamine, screening.

\section{INTRODUCTION}

Despite controversy about the potential for the development of dependence in relation to ecstasy [1], some people do experience problems with ecstasy use, and full case reports of dependence have been identified [2]. The Severity of Dependence Scale (SDS) is a brief, five-item screening instrument that has demonstrated validity in identifying cases of DSM cannabis, amphetamine, cocaine and benzodiazepine dependence. This study aimed to determine a cut-off score on the SDS that could provide a useful indication of individuals that are potentially ecstasy dependent according to DSM criteria.

\section{METHODS}

Interviews were conducted as part of the Ecstasy and Related Drugs Reporting System, a surveillance system for the ecstasy market [3]. A total of 198 participants were recruited through a purposive sampling strategy. Inclusion criteria required participants to be at least 16 , and to have used 'ecstasy' at least monthly in the preceding six months. Caseness (dependence) was assessed using the Composite International Diagnostic Interview (Short Form: CIDI-SF). This provided assessment of past-year DSM dependence symptoms, with the exception of withdrawal [4]. As some controversy exists over the status of the dependence syndrome for ecstasy [1], a conservative approach was adopted, whereby symptoms relating to lack of control over use (using in larger amounts or for longer than intended) and use interfering with role function were only scored positively if this occurred at least monthly in the preceding year. Diagnostic efficiency measures were calculated for each score on the SDS in identifying caseness.

\section{RESULTS}

The sample had a mean age of 23 years (range 17-47), and $57 \%(95 \% \mathrm{CI} 50-64)$ were male. Mean ecstasy use was 17 days of the past $180(\mathrm{SD}=14$, range 6-100). None were engaged in drug treatment. Area under the curve for the receiver operating characteristic was $0.76(95 \% \mathrm{CI}$ : $0.66-$ 0.85 ; nonparametric $\mathrm{p}<0.001$ ), suggesting that the SDS has good diagnostic utility. The Youden index (sensitivity + specificity -1) was used to identify an optimal cut-off, as the

*Address correspondence to this author at the School of Psychology, University of Tasmania, Private Bag 30, Hobart TAS 7001, Australia; Tel: +61 (3) 6226 2240; Fax: +61 (3) 62262883 ;

E-mail: Raimondo.Bruno@utas.edu.au maxima of this index represents the score that best balances sensitivity and specificity. This was apparent at an SDS value of 3 , which correctly classified $73 \%$ of cases, and identified $63 \%$ of true cases while ensuring that only $25 \%$ of real non-cases were screened positive. To examine validity of the identified cut-off, participants were categorised into negative $(\mathrm{SDS}<3 ; \mathrm{n}=135)$ and positive $(\mathrm{SDS} \geq 3 ; \mathrm{n}=63)$ groups. Univariate logistic regression models demonstrated that those screened positive consumed ecstasy more frequently, in larger amounts, and in binge patterns, and reported greater rates of social and role responsibility problems relating to use. Those screened positive were also more likely to drive under the influence of ecstasy, engage in high-risk alcohol use and experience clinical levels of psychological distress.

\section{DISCUSSION AND CONCLUSIONS}

Approximately one-fifth of this sample of regular ecstasy users were ecstasy dependent according to the DSM. This is comparable with studies of sentinel samples of ecstasy users internationally using different instruments [5-7]. This demonstrates that the experience of problems relating to ecstasy may be more common than would be expected on the basis of presentations to drug treatment - just 53 per million adult population in Australia in 2005/06, compared to 35,500 per million reporting recent ecstasy use [8] - and underscores the importance of screening for problems with use.

An SDS cut-off of 3 provides a good balance between sensitivity and specificity, and validly identifies individuals that are experiencing problems with their ecstasy use. For research applications, a more conservative cut-off (as high as 5) may be used to identify a more homogeneous group, but the lower cut-off is appropriate for clinical applications, particularly in light of the brevity of the SDS.

\section{REFERENCES}

[1] Degenhardt L, Bruno R, Topp L. Is ecstasy a drug of dependence? Drug Alcohol Depend 2010; 107: 1-10.

[2] Jansen K. Ecstasy (MDMA) dependence. Drug Alcohol Depend 1999; 53: 121-4.

[3] Topp L, Breen C, Kaye S, Darke S. Adapting the Illicit Drug Reporting System (IDRS) methodology to examine the feasibility of monitoring trends in party drug markets. Drug Alcohol Depend 2004; 73: 189-97.

[4] Kessler R, Andrews G, Mroczek D, Ustun B, Wittchen H. The World Health Organization Composite International Diagnostic Interview Short-Form (CIDI-SF). Int J Methods Psychiatr Res 1998; 7: 171-85. 
[5] Parsons J, Grov C, Kelly B. Club drug use and dependence among young adults recruited through time-space sampling. Public Health Reports 2009; 124: 246-54.

[6] Yen C, Hsu S. Symptoms of ecstasy dependence and correlation with psychopathology in Taiwanese adolescents. J Nerv Ment Dis 2007; 195: 866-9.
[7] Tossman P. The use of cannabis, ecstasy and amphetamines: is there a specific need for treatment? Sucht 2004; 50: 164-71.

[8] Degenhardt L, Roxburgh A, Dunn M, et al. The epidemiology of ecstasy use and harms in Australia. Neuropsychobiology 2009; 60: 176-87.

(C) Bruno et al.; Licensee Bentham Open.

This is an open access article licensed under the terms of the Creative Commons Attribution Non-Commercial License (http://creativecommons.org/licenses/ by-nc/3.0/) which permits unrestricted, non-commercial use, distribution and reproduction in any medium, provided the work is properly cited. 